\title{
Does Caffeine Citrate Administration Decrease Risk of Acute Kidney Injury in Hospitalized Preterm Neonates?
}

\author{
Fatma H. Mohamed*, Saneya A. Fahmy, Amal G. Mohamed \\ Department of Pediatrics, Faculty of Medicine for Girls Al-Azhar University, Cairo, Egypt \\ Email: ^fatmahanafy30@gmail.com
}

How to cite this paper: Mohamed, F.H., Fahmy, S.A. and Mohamed, A.G. (2021) Does Caffeine Citrate Administration Decrease Risk of Acute Kidney Injury in Hospitalized Preterm Neonates?. Open Journal of Pediatrics, 11, 532-542.

https://doi.org/10.4236/ojped.2021.113049

Received: August 30, 2021

Accepted: September 26, 2021

Published: September 29, 2021

Copyright $\odot 2021$ by author(s) and Scientific Research Publishing Inc. This work is licensed under the Creative Commons Attribution International License (CC BY 4.0).

http://creativecommons.org/licenses/by/4.0/

\begin{abstract}
Background: Acute kidney injury (AKI) is a life threatening condition with still high mortality rate in neonates. Early recognition of the risk factors and the rapid effective treatment of the contributing conditions will reduce AKI in the neonatal period. Aim of the Work: To determine association between early caffeine citrate administration and risk of acute kidney injury in hospitalized preterm neonates. Patients and Methods: This study was a prospective study that was conducted at the NICU of El Galaa Teaching Hospital. This study included 100 preterm neonates who were born alive from 32 weeks to 35 weeks of gestation. Results: There was highly statistically significant occurrence of apneaic episode in preterm babies who don't receive caffeine citrate and consequently decreased incidence of AKI in those babies. Conclusion: We concluded that caffeine citrate administration in preterm babies is associated with reduced incidence of apneaic episode and consequently reduced risk of AKI.
\end{abstract}

\section{Keywords}

Caffeine Citrate, Acute Kidney Injury, Neonates

\section{Introduction}

Acute kidney injury (AKI) is usually used to denote a sudden and often reversible reduction in the kidney function, as measured by glomerular filtration rate (GFR). Acute kidney injury (AKI) in the newborn is a common problem in the neonatal intensive care unit with many underlying risk factors such as asphyxia, respiratory distress syndrome (RDS), and sepsis [1].

The incidence of neonatal AKI is the highest one followed by adults and 
children, depending on different factors such as the gestational age, birth weight, perinatal asphyxia and sepsis [1].

Several different criteria have been used in research studies such as RIFLE

"Risk, Injury and Failure, Loss of kidney function and End-stage kidney disease", AKIN (Acute Kidney Injury Network) or KDIGO (Kidney Disease: Improving Global Outcomes) criteria [2].

However, KDIGO is the most recent and the clinically most commonly used. According to KDIGO, AKI is the presence of any of the following:

- Increase in serum creatinine by $\geq 0.3 \mathrm{mg} / \mathrm{dL}$ ( $\geq 26.5 \mathrm{micromol} / \mathrm{L}$ ) within 48 hours.

- Increase in serum creatinine to $\geq 1.5$ times baseline, within the prior seven days.

- Urine output $<0.5 \mathrm{~mL} / \mathrm{kg} / \mathrm{h}$ for at least six hours [2].

The etiology of AKI has always been traditionally divided into three categories:

- Pre-renal, renal and post-renal. Each of these categories has several different causes associated with them.

- Pre-renal causes include any reduced blood flow to the kidney. This may be part of a systemic hypoperfusion resulting from hypovolemia or hypotension [3].

- Renal causes include acute tubular necrosis which can result from several different causes. Prolonged renal ischemia, sepsis, and nephrotoxic drugs are the most common ones [4].

- Post-renal causes mainly include obstructive causes which lead to congestion of the filtration system and thus eventually lead to shutting down of the kidneys. The most common ones are renal/ureteral calculi, tumors or any urethral obstruction [5].

A preterm neonate is one who is born $<37$ weeks' gestation. Preterm babies are at increased risk of illness, disability and death [6].

Caffeine citrate is methylxanthine. It markedly reduces the number of apneic spells and the need for mechanical ventilation. The primary mechanism by which methylxanthines may decrease apnea is antagonism of adenosine, a neurotransmitter that can cause respiratory depression by blocking both its inhibitory A1 receptor and its excitatory A2A receptors. It is used with a loading dose of 20 $\mathrm{mg} / \mathrm{kg}$ of caffeine citrate $(10 \mathrm{mg} / \mathrm{kg}$ caffeine base) orally or intravenously $>30 \mathrm{mi}-$ nutes, followed by maintenance doses of 5 to $10 \mathrm{mg} / \mathrm{kg}$ in one daily dose beginning 24 hours after the loading dose [7].

\section{Aim of the Work}

The aim of the present study was to determine the association between early caffeine citrate administration and risk of acute kidney injury in hospitalized preterm neonates.

\section{Patients and Methods}

This study was a prospective study that was conducted at the NICU of El Galaa 
Teaching Hospital. This study included 100 preterm neonates who were born alive from 32 weeks to 35 weeks of gestation.

Full term neonates (born alive $>37$ weeks of gestation), neonates borne alive less than 32 weeks of gestation, neonates born alive more than 35 weeks of gestation, neonates with asphyxia, neonates with sepsis and neonates with major congenital anomalies were excluded from the study.

\subsection{Methodology}

1) Thorough history taking that focused on:

- Present history: Onset and duration of current NICU admission, and associated criteria related to AKI according to KDIGO (Kidney Disease: Improving Global Outcomes) criteria which include presence of any of the following:

- Increase in serum creatinine by $\geq 0.3 \mathrm{mg} / \mathrm{dL}$ ( $\geq 26.5 \mathrm{micromol} / \mathrm{L}$ ) within 48 hours.

- Increase in serum creatinine to $\geq 1.5$ times baseline, within the prior seven days.

- Urine output $<0.5 \mathrm{~mL} / \mathrm{kg} / \mathrm{h}$ for at least six hours [2].

Past history that included:

- Prenatal history: Maternal gravidity and parity and their outcomes, Conditions of medical importance during pregnancy such as bleeding, infections (that may contribute to perinatal asphyxia or neonatal sepsis) and prenatal exposures to nephrotoxic medications.

- Natal history: Gestational age, mode of delivery, maternal conditions that may contribute to perinatal asphyxia such as intrapartum bleeding and premature rupture of membranes.

- Postnatal history: Apgar scoring and NICU admission (onset and duration).

- Family history: History of renal diseases in family, history of sibling death and consanguinity.

\section{2) Thorough clinical examination including:}

Anthropometrics (weight, length and head circumference), hydration state and urine output during NICU admission, symptoms and signs of AKI according to KDIGO criteria [2].

3) Thorough laboratory investigations that included:

Blood urea nitrogen (BUN), Serum creatinine, Sodium, Potassium, Calcium, Complete blood count, C-reactive protein, Blood culture, Arterial blood gases on admission with follow up every week, for cases of AKI laboratory investigations were repeated daily.

\subsection{Ethical Consideration}

- An informed oral and written consent was obtained from all parents of children to participate in the study.

- The steps of study, the aim, the potential benefits all were discussed with parents of the patients.

- All participants had the right to withdraw from the study at any time without giving reasons. 


\subsection{Statistical Analysis}

Data were collected, revised, coded and entered to the Statistical Package for Social Science (IBM SPSS) version 20. The qualitative data were presented as number and percentages while quantitative data were presented as mean, standard deviations and ranges when their distribution was found parametric.

The comparison between two groups with qualitative data was done by using Chi-square test and/or Fisher exact test was used instead of Chi-square test when the expected count in any cell was found less than 5 .

The comparison between two independent groups with quantitative data and parametric distribution was done by using Independent t-test.

The confidence interval was set to $95 \%$ and the margin of error accepted was set to $5 \%$. So, the $\mathrm{p}$-value was considered significant as the following:

- $\mathrm{P}>0.05=$ non significant (NS);

- $\mathrm{P}<0.05=$ significant $(\mathrm{S})$;

- $\mathrm{P}<0.001=$ highly significant (HS).

\section{Results}

Table 1 shows that there was highly statistically significant increase of urea and creatinine regarding preterm neonates who don't receive caffeine citrate in comparison to preterm neonates who received caffeine citrate.

Table 2 shows that there was highly statistically significant difference between two groups regarding $\mathrm{CBC}$.

Table 3 shows that there was highly statistically significant occurrence of ABG parameters $\mathrm{PH}$ (on admission), $\mathrm{PO}_{2}$ (on admission), $\mathrm{PCO}_{2}$ (on admission), $\mathrm{pH}$ (follow up), $\mathrm{PCO}_{2}$ (follow up), $\mathrm{PO}_{2}$ (follow up) and $\mathrm{HCO}_{3}$ (follow up) regarding preterm neonates who don't receive caffeine citrate in comparison with preterm neonates who received caffeine citrate, and there was no statistically significant difference between two groups regarding $\mathrm{HCO}_{3}$ (on admission).

Table 4 shows that there was highly statistically significant increase of mean value regarding $\mathrm{Na}$ and $\mathrm{Ca}$ of preterm neonates who don't receive caffeine citrate, and there was no statistically significant difference between two groups regarding $\mathrm{K}$.

Table 1. Comparison between received caffeine citrate $($ no. $=50)$ and not received caffeine citrate (no. $=50)$ regarding AKI criteria.

\begin{tabular}{cccccc}
\hline & \multicolumn{4}{c}{ Received caffeine citrate } & \multirow{2}{*}{ P value• } \\
\cline { 2 - 5 } & \multicolumn{3}{c}{ Yes } & No & \\
\cline { 2 - 5 } & Mean & SD & Mean & SD & \\
\hline Urea (D2) & 19.18 & 7.53 & 27.92 & 8.45 & $<0.001$ \\
Creat (D2) & 0.81 & 0.30 & 1.08 & 0.24 & $<0.001$ \\
Urea (D7) & 11.54 & 3.69 & 20.66 & 8.01 & $<0.001$ \\
Creat (D7) & 0.53 & 0.19 & 0.85 & 0.26 & $<0.001$ \\
\hline
\end{tabular}

P-value $>0.05$ : No significant; P-value $<0.05$ : Significant; P-value $<0.01$ : highly significant. *: Chi-square test, $\bullet$ : Independent $\mathrm{t}$-test. 
Table 2. Comparison between received caffeine citrate $($ no. $=50)$ and not received caffeine citrate (no. $=50)$ regarding $\mathrm{CBC}$.

\begin{tabular}{|c|c|c|c|c|c|}
\hline & \multicolumn{4}{|c|}{ Received caffeine citrate } & \multirow{3}{*}{$P$ value. } \\
\hline & \multicolumn{2}{|c|}{ Yes } & \multicolumn{2}{|c|}{ No } & \\
\hline & Mean & SD & Mean & SD & \\
\hline $\mathrm{Hb}$ & 16.36 & 2.00 & 16.23 & 1.89 & 0.750 \\
\hline Hct & 49.62 & 7.01 & 49.89 & 6.81 & 0.849 \\
\hline TLC & 13.18 & 4.83 & 13.05 & 4.79 & 0.889 \\
\hline Platelets & 252.99 & 68.29 & 265.81 & 75.89 & 0.377 \\
\hline
\end{tabular}

P-value $>0.05$ : No significant; P-value $<0.05$ : Significant; P-value $<0.01$ : highly significant. *: Chi-square test, $\bullet$ : Independent t-test.

Table 3. Comparison between received caffeine citrate $($ no. $=50)$ and not received caffeine citrate (no. $=50)$ regarding $\mathrm{ABG}$

\begin{tabular}{|c|c|c|c|c|c|}
\hline & \multicolumn{4}{|c|}{ Received caffeine citrate } & \multirow{3}{*}{$P$ value• } \\
\hline & \multicolumn{2}{|c|}{ Yes } & \multicolumn{2}{|c|}{ No } & \\
\hline & Mean & SD & Mean & SD & \\
\hline $\mathrm{PH}$ (on admission) & 7.27 & 0.05 & 7.24 & 0.05 & 0.005 \\
\hline $\mathrm{PO}_{2}$ (on admission) & 37.80 & 9.43 & 31.74 & 10.30 & 0.003 \\
\hline $\mathrm{PCO}_{2}$ (on admission) & 44.78 & 11.38 & 51.10 & 7.40 & 0.001 \\
\hline $\mathrm{HCO}_{3}$ (on admission) & 20.80 & 3.43 & 21.52 & 2.79 & 0.252 \\
\hline PH (Follow up) & 7.36 & 0.05 & 7.31 & 0.04 & $<0.001$ \\
\hline $\mathrm{PCO}_{2}$ (Follow up) & 39.46 & 3.47 & 45.36 & 7.28 & $<0.001$ \\
\hline $\mathrm{PO}_{2}$ (Follow up) & 40.84 & 7.94 & 37.12 & 7.42 & 0.017 \\
\hline $\mathrm{HCO}_{3}$ (Follow up) & 22.98 & 1.90 & 24.06 & 1.90 & 0.005 \\
\hline
\end{tabular}

$\mathrm{P}$-value $>0.05$ : No significant; P-value $<0.05$ : Significant; P-value $<0.01$ : highly significant. ${ }^{*}$ : Chi-square test, $\bullet$ : Independent t-test.

Table 4. Comparison between received caffeine citrate $($ no. $=50)$ and not received caffeine citrate (no. $=50)$ regarding $\mathrm{Na}, \mathrm{K}$ and $\mathrm{Ca}$.

\begin{tabular}{cccccc}
\hline & \multicolumn{4}{c}{ Received caffeine citrate } & \multirow{2}{*}{ P value• } \\
\cline { 2 - 5 } & \multicolumn{3}{c}{ Yes } & No & \\
\cline { 2 - 5 } & Mean & SD & Mean & SD & 0.001 \\
$\mathrm{Na}$ & 133.44 & 2.69 & 135.08 & 1.88 & 0.328 \\
$\mathrm{~K}$ & 4.89 & 0.56 & 4.80 & 0.38 & $<0.001$ \\
$\mathrm{Ca}$ & 7.28 & 1.20 & 8.08 & 0.76 &
\end{tabular}

P-value $>0.05$ : No significant; P-value $<0.05$ : Significant; P-value $<0.01$ : highly significant. ${ }^{*}$ : Chi-square test, $\bullet$ : Independent t-test.

Table 5 shows that there was highly statistically significant occurrence of apneaic episode in preterm neonates who don't receive caffeine citrate in comparison to preterm babies who received caffeine citrate.

Table 6 shows that there was no statistically significant difference between two groups regarding sex and method of delivery. 
Table 5. Comparison between received caffeine citrate $($ no. $=50)$ and not received caffeine citrate (no. $=50)$ regarding apnea.

\begin{tabular}{|c|c|c|c|c|c|c|}
\hline & & \multicolumn{4}{|c|}{ Received caffeine citrate } & \multirow{3}{*}{$P$ value } \\
\hline & & \multicolumn{2}{|c|}{ Yes } & \multicolumn{2}{|c|}{ No } & \\
\hline & & No & $\%$ & No & $\%$ & \\
\hline \multirow{2}{*}{ Apnea } & Yes & 10 & $20.0 \%$ & 40 & $80.0 \%$ & \multirow{2}{*}{$<0.001^{*}$} \\
\hline & No & 40 & $80.0 \%$ & 10 & $20.0 \%$ & \\
\hline
\end{tabular}

P-value $>0.05$ : No significant; P-value $<0.05$ : Significant; P-value $<0.01$ : highly significant. ${ }^{*}$ : Chi-square test, $\bullet$ : Independent t-test.

Table 6. Comparison between received caffeine citrate (no. $=50)$ and not received caffeine citrate (no. $=50)$ regarding sex.

\begin{tabular}{|c|c|c|c|c|c|c|}
\hline & & \multicolumn{4}{|c|}{ Received caffeine citrate } & \multirow{3}{*}{$P$ value } \\
\hline & & \multicolumn{2}{|c|}{ Yes } & \multicolumn{2}{|c|}{ No } & \\
\hline & & No & $\%$ & No & $\%$ & \\
\hline \multirow{2}{*}{ Sex } & Male & 23 & $46.0 \%$ & 24 & $48.0 \%$ & \multirow{2}{*}{$0.841^{*}$} \\
\hline & Female & 27 & $54.0 \%$ & 26 & $52.0 \%$ & \\
\hline
\end{tabular}

In recent decades, advances in the care of preterm neonates have dramatically lowered mortality rates [2]. As survival has improved, clinicians have been increasingly focused on decreasing the short-term and long-term sequelae of prematurity [8].

Although AKI is independently associated with increased morbidity and mortality [4], specific therapies remain elusive. To date, the only medication shown in randomized clinical trials to ameliorate AKI in neonates is the adenosine antagonist theophylline [9].

This medication is not widely used due to concerns regarding its narrow therapeutic index and potential toxicity, particularly in premature neonates. However, a different adenosine antagonist-caffeine-is commonly used in modern neonatal care for treatment of apnea of prematurity [10].

Although no randomized trial has evaluated caffeine in preventing or treating AKI, two recent observational studies have demonstrated an inverse association between caffeine exposure and AKI occurrence [11] [12].

There are several potential mechanisms of action through which caffeine and possibly other methylxanthines could directly reduce AKI: 1) Increased renal blood flow, enhanced sodium excretion, and a higher glomerular filtration rate. 2) Caffeine citrate counteracts the hypoxemia-induced renal hemodynamic changes by maintaining renal vascular resistance. 3) Another potential mechanism of caffeine-mediated renal protection may involve attenuation of oxidative stress and injury on endoplasmic reticulum [12].

Caffeine citrate administration in preterm neonate decrease occurrence of apnea and the risk of hypoxia so, will decrease risk of prerenal injury and decrease risk of AKI. 
The aim of this study was to determine association between early caffeine citrate administration and risk of acute kidney injury in hospitalized preterm neonates.

To obtain this aim, we included 100 preterm neonates born alive from 32 weeks to 35 weeks of gestation and with AKI according to KDIGO (Kidney Disease: Improving Global Outcomes) criteria [2], hospitalized in NICU of El Galaa Teaching Hospital. 47.0\% of our cases were males, mean gestational age $33.21 \pm$ 1.19 weeks, mean birth weight was $1.69 \pm 0.45 \mathrm{~kg} \&$ the mean birth weight was $(1.69 \mathrm{~kg} \pm 0.45 \mathrm{~kg})$ ranging between $(1.1 \mathrm{~kg}$ to $3.1 \mathrm{~kg})$.

The AWAKEN study [12] who studied 675 cases was $55.4 \%$ of them were male, with a mean (SD) gestational age of 28.9 (2.8) weeks and a mean (SD) birth weight of 1285 (477) g.

In Felipin et al. [13], who observed that the prevalence of AKI was more present in extreme preterm infants, that is, less than 28 weeks of gestational age and in PTNB less than 1500 grams like our study results.

Low birth weight is defined as birth weight of less than 2, 500 grams, which may result from Intrauterine Growth Restriction and/or preterm birth. Low birth weight presents with difficulties in the digestion process and lose more weight in the first days of life due to the lack of appropriate nutritional intake due to the immaturity of the digestive system. NB with low birth weight and gestational age less than 32 weeks present a high risk of renal diseases due to the reduced number of nephrons [14].

At Day 2 after admission, mean urea among our cases was $27.49 \pm 16.90$ $\mathrm{mg} / \mathrm{Dl} \&$ Mean creatinine was $0.82 \pm 0.27 \mathrm{mg} / \mathrm{dL}$. While at Day 7 after admission, mean urea was $15.39 \pm 7.48 \mathrm{mg} / \mathrm{dL} \&$ Mean creatinine was $0.53 \pm 0.19$ $\mathrm{mg} / \mathrm{dL}$.

Acute kidney injury occurred in 122 neonates (18.1\%) in the first 7 days after birth in $A W A K E N$ study [12].

Renal flow is considerably reduced in preterm infants, which increases the chance of this population presenting AKI as a result of renal ischemia [15] [16] [17]. It should be emphasized that renal immaturity, from the anatomical and physiological point of view, makes extra uterine life difficult, sometimes hampered by malnutrition, drug nephrotoxicity and other complications present during hospitalization [10].

The field of neonatal AKI has undergone a renaissance, in numerous studies Harer et al. [12], Srinivasan et al. [18] and Jetton et al. [19] like our study results demonstrating that AKI in critically ill neonates is common and consistently associated with adverse outcomes.

Mean $\mathrm{Hb}$ among cases in this study was $16.29 \pm 1.94 \mathrm{~g} / \mathrm{dl}$, mean Hctwas $49.75 \% \pm 6.88 \%$, mean Total leucocytic count was $13.11 \pm 4.79$ cells per liter, while mean platelet count was $262.69 \pm 64.2910^{9} / \mathrm{L}$.

On admission of our studied cases, mean $\mathrm{PH}$ was $7.28 \pm 0.17$, mean $\mathrm{PO}_{2}$ was $49.23 \pm 14.64$ torr, mean $\mathrm{PCO}_{2}$ was $45.82 \pm 15.79$ torr \& mean $\mathrm{HCO}_{3}$ was $22.24 \pm$ 
$4.83 \mathrm{mEq} / \mathrm{L}$. While at Follow up, mean $\mathrm{PH}$ was $7.33 \pm 0.16$, mean $\mathrm{PCO}_{2}$ was $44.99 \pm 13.08$ torr, mean $\mathrm{PO} 2$ was $45.14 \pm 14.14$ torr $\&$ mean $\mathrm{HCO}_{3}$ was $23.19 \pm$ $3.90 \mathrm{mEq} / \mathrm{L}$.

Among our studied cases, $50 \%$ received caffeine citrate \& $50.0 \%$ didn't receive caffeine citrate.

Of the 675 participants included in Harer et al. [12] study, 447 (66.2\%) received at least one dose of caffeine in the first 7 days after birth and before AKI.

While Caffeine exposure occurred in $72.1 \%$ of all patients included in Carmody et al. [11] study and $54.5 \%$ of those who received prolonged invasive respiratory support.

According to our study, there was highly significant statistically occurance in preterm babies regarding both uraea \& creatinine in day 2 , both urea \& creatinine in day 7 . But there was significant statistical occurance between groups regarding birth weight $(\mathrm{P}=0.029)$.

In contrary to our study, in Harer et al. [12] who studied Neonates who did not receive caffeine had older gestational age, higher birth weight, greater Apgar scores at 5 minutes, suggesting that these neonates were not sicker than those who received caffeine.

In contrary to our result, $A W A K E N$ study [12] reported that Acute kidney injury occurred less frequently among neonates who received caffeine than among those who did not (50 of 447 [11.2\%] vs 72 of 228 [31.6\%], P < 0.01). After multivariable adjustment, administration of caffeine remained associated with reduced odds of developing AKI (adjusted odds ratio, 0.20; 95\% CI, 0.11 0.34 ), indicating that for every 4.3 neonates exposed to caffeine one case of AKI was prevented. Among neonates with early AKI, those receiving caffeine were less likely to develop stage 2 or 3 AKI (adjusted odds ratio, 0.20; 95\% CI, 0.12 $0.34)$.

In contrary to our study results, Neonates who received caffeine in Harer et al. [12] who studied were less likely to develop early AKI compared with those who did not $(\mathrm{P}<0.01)$. Neonates who received caffeine were less likely to develop early AKI based on sCr values alone (adjusted OR, 0.20; 95\% CI, 0.11 - 0.37); however, there was no association of use of caffeine with early AKI as defined by decreased Urine OutPut without a rise in creatinine (adjusted OR, 0.40; 95\% CI, 0.15 - 1.06). Similar associations were observed for AKI that occurred after the initial 7 days (before NICU discharge, transfer, death, or age 120 days). The incidence of AKI through the entire data collection period was $27.6 \%$ (186 of 675). Among patients receiving caffeine, the odds of developing AKI were reduced by $44 \%$ during the data collection period (crude OR, 0.56; 95\% CI, $0.38-0.84$ ).

In discordance with our result, Carmody et al. [11] who reported that AKI occurred less frequently in neonates who received caffeine (all patients: $17.8 \%$ vs $43.6 \% ; \mathrm{P}=0.002$; prolonged invasive respiratory support: $29.2 \%$ vs $75.0 \%$; $\mathrm{P}=$ 0.002). Also reported that Caffeine exposure was associated with decreased odds for AKI in logistic regression models adjusted for sex, birth weight, gestational 
age, small for gestational age status, illness severity on admission, and receipt of indomethacin, invasive ventilation, dopamine, aminoglycosides, and vancomycin (all patients: OR 0.22; 95\% CI $0.07-0.75, \mathrm{P}=0.02$; prolonged invasive respiratory support subgroup: OR 0.06; 95\% CI $0.01-0.57, \mathrm{P}=0.02$ ).

Hare et al., Carmody et al., obtained significant relation between caffeine citrate and AKI may be due to large sample size.

In our study, $\mathrm{PH}$ on admission was significantly higher among patients who received caffeine citrate when compared with patients who didn't $(\mathrm{P}=0.032)$. While $\mathrm{PO}_{2}, \mathrm{PCO}_{2} \& \mathrm{HCO}_{3}$ at Follow up were significantly lower among patients who received caffeine citrate when compared with patients who didn't $(\mathrm{P}=$ 0.001 for $\mathrm{PCO}_{2} \& \mathrm{P}<0.001$ for $\mathrm{PO}_{2} \& \mathrm{HCO}_{3}$ ).

On admission, those receiving caffeine in Harer et al. [12] study were more likely to have a diagnosis of respiratory distress, undergo a sepsis evaluation, and receive either invasive or noninvasive respiratory support.

According to the present study, there was no significant statistical occurrence regarding both groups regarding $\mathrm{HCO}_{3}$ at Follow up, $\mathrm{PH} \& \mathrm{PCO}_{2}$ at Follow up were significantly lower among patients who received caffeine citrate when compared with patients who didn't $\left(\mathrm{P}=0.002\right.$ for $\mathrm{PH} \& \mathrm{P}<0.001$ for $\left.\mathrm{PCO}_{2}\right)$. While $\mathrm{PO}_{2}$ at Follow up was significantly higher among patients who received caffeine citrate when compared with patients who didn't $(\mathrm{P}=0.029)$.

In very low birth weight infants, caffeine is used almost doubled between 1997 and 2010 , from $40 \%$ to $70 \%$ [10]. This dramatic increase coincides with clinical trials, such as the Caffeine for Apnea of Prematurity Trial, in which infants randomized to early treatment with caffeine were less likely to develop bronchopulmonary dysplasia.

Age at initiation of caffeine administration fell from a mean of 10 to 12 days after birth (median, 4 - 5 days) to a mean of 4 days (median, 1 day). In addition, the early use of caffeine has been associated with significant decreases in early-onset and late-onset sepsis, pulmonary interstitial emphysema, intra ventricular hemorrhage, retinopathy of prematurity, duration of mechanical ventilation, bronchopulmonary dysplasia, and treatment of patent ductus arteriosus [10]. Given these data, it is possible that decreased frequency of AKI among infants receiving caffeine is not mediated by any direct association of caffeine with kidney function but is instead conferred through benefits in neonatal respiratory status or hemodynamic stability.

\section{Conclusion}

This study found that caffeine citrate administration in preterm infants is associated with reduced incidence of apneaic episode in preterm neonates and consequently decreased risk and severity of AKI.

\section{Conflicts of Interest}

The authors declare no conflicts of interest regarding the publication of this paper. 


\section{References}

[1] Ghobrial, E.E., Elhouchi, S.Z., Eltatawy, S.S. and Beshara, L.O. (2018) Risk Factors Associated with Acute Kidney Injury in Newborns. Saudi Journal of Kidney Disease and Transplantation, 29, 81-87. https://doi.org/10.4103/1319-2442.225179

[2] Bıyık, M., Ataseven, H., Bıyık, Z., Asil, M., Çifçi, S., Sayın, S., Demir, A. and Tombul, H.Z. (2016) KDIGO (Kidney Disease: Improving Global Outcomes) Criteria as a Predictor of Hospital Mortality in Cirrhotic Patients. The Turkish Journal of Gastroenterology, 27, 173-179. https://doi.org/10.5152/tjg.2016.15467

[3] Favre, N., Burnier, M. and Kissling, S. (2016) When the Nephrologist Should Be Called in the Emergency Room. Revue Médicale Suisse, 12, 398-403.

[4] Moledina, D.G. and Perazella, M.A. (2016) PPIs and Kidney Disease: from AIN to CKD. Journal of Nephrology, 29, 611-616. https://doi.org/10.1007/s40620-016-0309-2

[5] Meola, M., Nalesso, F., Petrucci, I., Samoni, S. and Ronco, C. (2016) Pathophysiology and Clinical Work-Up of Acute Kidney Injury. Contributions to Nephrology, 188, 1-10. https://doi.org/10.1159/000445460

[6] Vohr, B. (2013) Long-Term Outcomes of Moderately Preterm, Late Preterm and Early Term Infants. Clinics in Perinatology, 39, 769-776.

https://doi.org/10.1016/j.clp.2013.07.006

[7] Eichenwald, E.C. and the Committee on Fetus and Newborn (2016) Apnea of Prematurity. Pediatrics, 137, 1-7. https://doi.org/10.1542/peds.2015-3757

[8] Patel, B.K. and Shah, J.S. (2012) Necrotizing Enterocolitis in Very Low Birth Weight Infants: A Systemic Review. ISRN Gastroenterology, 2012, Article ID: 562594.

https://doi.org/10.5402/2012/562594

[9] Raina, A., Pandita, A., Harish, R., Yachha, M. and Jamwal, A. (2016) Treating Perinatal Asphyxia with Theophylline at Birth Helps to Reduce the Severity of Renal Dysfunction in Term Neonates. Acta Paediatrica, 105, e448-e451.

https://doi.org/10.1111/apa.13469

[10] Dobson, N.R., Patel, R.M., Smith, P.B., et al. (2014) Trends in Caffeine Use and Association between Clinical Outcomes and Timing of Therapy in Very Low Birth Weight Infants. The Journal of Pediatrics, 164, 992-998.e3. https://doi.org/10.1016/j.jpeds.2013.12.025

[11] Carmody, J.B., Harer, M.W., Denotti, A.R., Swanson, J.R. and Charlton, J.R. (2016) Caffeine Exposure and Risk of Acute Kidney Injury in a Retrospective Cohort of Very Low Birth Weight Neonates. The Journal of Pediatrics, 172, 63-68.e1. https://doi.org/10.1016/j.jpeds.2016.01.051

[12] Harer, M.W., Askenazi, D.J., Boohaker, L.J., Carmody, J.B., Griffin, R.L., Guillet, R., Selewski, D.T., Swanson, J.R. and Charlton, J.R. (2018) Association between Early Caffeine Citrate Administration and Risk of Acute Kidney Injury in Preterm Neonates: Results from the AWAKEN Study. JAMA Pediatrics, 172, e180322. https://doi.org/10.1001/jamapediatrics.2018.0322

[13] Felipin, L.C.S., Oliveira, R.R., Merino, M.F.G.L., Rodrigues, B.C. and Higarashi, I.H. (2019) Associated Factors for Acute Kidney Injury in Preterm Infants. Revista Brasileira de Enfermagem, 72, 118-124. https://doi.org/10.1590/0034-7167-2018-0231

[14] Zohdi, V., Sutherland, M.R., Lim, K., Gubhaju, L., Zimanyi, M.A. and Black, M.J. (2012) Low Birth Weight Due to Intrauterine Growth Restriction and/or Preterm Birth: Effects on Nephron Number and Long-Term Renal Health. International Journal of Nephrology, 2012, Article ID: 136942. 
https://doi.org/10.1155/2012/136942

[15] Bansal, S.C., Nimbalkar, A.S., Kungwani, A.R., Patel, D.V., Sethi, A.R. and Nimbalkar, S.M. (2017) Clinical Profile and Outcome of Newborns with Acute Kidney Injury in a Level 3 Neonatal Unit in Western India. Journal of Clinical and Diagnostic Research, 11, SC01-SC04.

[16] Selewski, D.T., Jordan, B.K., Askenazi, D.J., Dechert, R.E. and Sarkar, S. (2013) Acute Kidney Injury in Asphyxiated Newborns Treated with Therapeutic Hypothermia. The Journal of Pediatrics, 162, 725-729.

https://doi.org/10.1016/j.jpeds.2012.10.002

[17] Nagaraj, N., Berwal, P.K., Srinivas, A. and Berwal, A. (2016) A Study of Acute Kidney Injury in Hospitalized Preterm Neonates in NICU. Journal of Neonatal-Perinatal Medicine, 9, 417-421. https://doi.org/10.3233/NPM-161614

[18] Srinivasan, N., Schwartz, A., John, E., Price, R. and Amin, S. (2018) Acute Kidney Injury Impairs Postnatal Renal Adaptation and Increases Morbidity and Mortality in Very Low-Birth-Weight Infants. The American Journal of Perinatology, 35, 39-47. https://doi.org/10.1055/s-0037-1604470

[19] Jetton, J.G., Guillet, R. and Askenazi, D.J. (2016) Assessment of Worldwide Acute Kidney Injury Epidemiology in Neonates: Design of a Retrospective Cohort Study. Frontiers in Pediatrics, 4, 68. https://doi.org/10.3389/fped.2016.00068 